Research

\title{
Multimorbidity and quality of life: a closer look
}

\author{
Martin Fortin*1,2, Marie-France Dubois ${ }^{3,4}$, Catherine Hudon ${ }^{1,2}$, \\ Hassan Soubhi ${ }^{1,2}$ and José Almirall ${ }^{2}$
}

\begin{abstract}
Address: ${ }^{1}$ Department of Family Medicine, Sherbrooke University, Sherbrooke, Canada, ${ }^{2}$ Unité de médecine de famille, Centre de Santé et de services sociaux de Chicoutimi, Chicoutimi, Canada, ${ }^{3}$ Department of Community Health Sciences, Sherbrooke University, Sherbrooke, Canada and ${ }^{4}$ Research Center on Aging, Sherbrooke Geriatric University Institute, Canada

Email: Martin Fortin* - Martin.Fortin@USherbrooke.ca; Marie-France Dubois - Marie-France.Dubois@USherbrooke.ca; Catherine Hudon - Catherine.Hudon@USherbrooke.ca; Hassan Soubhi - Hassan.Soubhi@USherbrooke.ca;

José Almirall - Jose.Almirall@USherbrooke.ca

* Corresponding author
\end{abstract}

Published: 6 August 2007

Health and Quality of Life Outcomes 2007, 5:52 doi:10.1186/1477-7525-5-52
Received: 4 May 2007

Accepted: 6 August 2007

This article is available from: http://www.hqlo.com/content/5/I/52

(C) 2007 Fortin et al; licensee BioMed Central Ltd.

This is an Open Access article distributed under the terms of the Creative Commons Attribution License (http://creativecommons.org/licenses/by/2.0), which permits unrestricted use, distribution, and reproduction in any medium, provided the original work is properly cited.

\begin{abstract}
Background: The presence of multiple chronic conditions is associated with lower health related quality of life (HRQOL). Disease severity also influences HRQOL. To analyse the effects of all possible combinations of single diseases along with their severity on HRQOL seems cumbersome. Grouping diseases and their severity in specific organ domains may facilitate the study of the complex relationship between multiple chronic conditions and HRQOL. The goal of this study was to analyse impaired organ domains that affect the most HRQOL of patients with multiple chronic conditions in primary care and their possible interactions.
\end{abstract}

Methods: We analysed data from 238 patients recruited from the clientele of 21 family physicians. We classified all chronic conditions along with the measure of their severity into the 14 organ domains of the Cumulative Illness Rating Scale (CIRS). Patients also completed the 36-item Medical Outcomes Study questionnaire (SF-36). One-way analyses of variance were performed to study the relationship between the severity score for each CIRS domain and both physical component summary (PCS) and mental component summary (MCS) of HRQOL. Two-way analyses of variance were conducted to investigate the significance of possible organ domains interactions. Variables involved in significant bivariate relationships or interactions were candidates for inclusion in a multivariate model. Five additional variables were included in the multivariate model because of their possible confounding effect: perceived social support, age, education, perceived economic status and residual CIRS.

Results: Significant differences in the PCS $(p<0.01)$ were found in 12 of the 14 CIRS organ domains. A significant difference in MCS was found only in the Psychiatric domain. In the multivariate analysis for the PCS, the CIRS domains Musculoskeletal, Neurological, and Psychiatric, had an independent direct impact on PCS while the Upper gastrointestinal, Vascular, Cardiac and Respiratory domains were involved in interactions. A multivariate model was not necessary for the mental component.

Conclusion: Vascular, Upper gastrointestinal and Musculoskeletal systems have strong negative effects on HRQOL. Among combinations of systems, the respiratory and cardiac combination is of particular concern because of a synergistic negative effect. This study paves the way for a future study with a bigger sample that could yield a model of wider generalizability. 


\section{Background}

Goals of caring for patients with a chronic condition are to enhance their functional status, minimize symptoms, control pain, reduce disability, and prolong life through secondary prevention [1]. Patients with multiple chronic conditions or multimorbidity are the rule rather than the exception in primary care [2]. For family physicians to pursue the same care goals for patients with multimorbidity, they have to develop a partnership with patients and set priorities to address patients' health care needs with the aim of increasing their life span while maintaining an optimal quality of life $[3,4]$.

Health-related quality of life (HRQOL) provides a multidimensional perspective that encompasses a patient's physical, emotional, and social functioning [5]. In general, patients with more than one comorbid condition report the poorest level of HRQOL [6], but some chronic conditions are more strongly associated with poor HRQOL than others. The severity of the individual conditions is also a factor influencing HRQOL[6]. Knowledge of the conditions that potentially have a greater impact on the HRQOL may help family physicians focus their attention on those conditions. The combined influence of pairs of chronic medical conditions on HRQOL have received some attention, [7-10] but patients with multimorbidity often present more than two chronic medical conditions and have often multiple body systems involved [2].

Disease interactions known to affect HRQOL include, among others, diabetes with hypertension, heart disease, arthritis, obesity and respiratory problems; arthritis with heart disease, hypertension, pulmonary diseases and obesity [7-10]. To make an analysis using individually all possible chronic conditions, their possible combinations and effects on the HRQOL seems cumbersome. Grouping diseases and their severity in specific organ domains may facilitate the understanding of the complex relationship between multiple chronic conditions and HRQOL. We used the Cumulative Illness Rating Scale (CIRS) in a previous study to document the association between HRQOL and multimorbidity (presence of two or more chronic conditions). We showed that the CIRS was a better predictor of HRQOL than a simple count of chronic diseases [6]. The CIRS is a comprehensive comorbidity index which takes into account the severity of the conditions using a scoring mechanism that encompasses 14 anatomical domains (Cardiac, Vascular, Hematological, Respiratory, Ophtalmological-ORL, Upper gastrointestinal, Lower gastrointestinal, Hepatic-pancreatic, Renal, Genitourinary, Musculoskeletal-tegumental, Neurological, Endocrinemetabolic-breast, and Psychiatric) [11-14]. The total CIRS score is the sum of severity scores assigned to each organ domain. So far, we have only used the total CIRS score in our studies $[6,15,16]$. We hypothesize that the individual organ domain score may further our understanding of the complex relationship between multimorbidity and HRQOL.

The goal of this study was therefore to analyse which impaired organ domains affect the most HRQOL of patients with multiple chronic conditions in primary care and their possible interactions.

\section{Methods}

We used data collected in a group of 238 adult primary care patients (age 18 or older) recruited from the clientele of 21 family physicians in the Saguenay region, Canada, who participated in a study on HRQOL [6]. Details of the selection process are described elsewhere [6]. In brief, we randomly selected patients from 980 patients who had also been selected at random for a prevalence study on multimorbidity [2]. Our goal was to recruit patients to complete five CIRS groups (quintiles) of 60 patients each with different levels of multimorbidity. Of the 419 patients we tried to contact by phone, 66 could not be reached, despite repeated attempts. Of the remaining 353 patients, 238 agreed to participate (participation rate of $67 \%)$. Patients who refused to participate did not have time (66 patients), or were not interested (42 patients); 7 patients were judged to be in an acute state of illness and were not included.

From an exhaustive chart review, we extracted a comprehensive list of diagnoses of all chronic conditions for every patient after informed consent. For this review, the information contained in the medical chart was considered valid and complete. We then scored the Cumulative Illness Rating Scale (CIRS) [17].

Each domain of the CIRS is scored from 0 to 4 as follows: 0 , no problem; 1 , minor current problem or significant history; 2, morbidity or moderate discomfort, requiring treatment; 3 , severe problem, constant significant discomfort, chronic problem difficult to control; 4, extremely severe problem, requiring immediate treatment, organ failure or severe functional impairment. The theoretical score ranges from 0 to 56 , but scores close to the highest value are incompatible with life. The CIRS has been shown to be valid and reliable for its use in primary care [18].

Patients completed the French version of the self-administered 36-item short form of the Medical Outcomes Study questionnaire (SF-36) that is well known and validated to assess HRQOL [19]. The SF-36 comprises 8 multi-item scales divided into 2 main groups: physical and mental aspects of quality of life. Two summary scores for each group are obtained through a weighted sum of these scales. For all scales and both summary scales, lower 
scores indicate lower HRQOL. In this study, we used the physical component summary (PCS) and the mental component summary (MCS) in the analyses.

Data for potential confounders (age, education, self-perceived economic status and self-perceived social support) were also collected. Self-perceived social support was measured with the Social Provisions Scale [20].

The research ethics board of the Centre de santé et de services sociaux de Chicoutimi approved this study.

\section{Statistical analysis}

Characteristics of the participants were first summarized using means and standard deviations or percentages. We then examined the proportion of subjects falling into each severity level for the 14 domains covered by the CIRS. Severity levels with low prevalence (less than 10 subjects) were combined with the adjacent level for subsequent analyses.

For each domain of the CIRS, one-way analyses of variance (ANOVA) were first performed to study the relationship between severity and both physical (PCS) and mental (MCS) components of quality of life, ignoring the effect of other domains. Severity levels with non significant differences in quality of life were combined. Two-way analyses of variance were conducted to investigate the significance of possible interactions based on disease interactions previously identified in the literature [7-10] or in our practice. The CIRS domains included in the analysis of interactions for the PCS were: Cardiac, Vascular, Respiratory, Upper gastrointestinal, Musculoskeletal, Neurological, Endocrine. Variables involved in bivariate relationships or interactions significant at the 0.15 level were candidates for inclusion in a multivariate model.

Five additional variables were included in the multivariate model because of their possible confounding effect: perceived social support, age, education, perceived economic status and residual CIRS [6]. The latter was defined as the sum of severity scores for the systems excluded from the model. A backward elimination procedure was then applied to eliminate variables which cease to be statistically significant $(p>0.05)$ in the presence of others. However, variables with a significant interaction term were kept in the model, regardless of their significance level to ensure the hierarchy principle. Analyses were performed with SAS (SAS Institute Inc., Cary, NC), version 8.02.

\section{Results}

Characteristics of the 238 participants are reported elsewhere [6]. On average, participants were $59.0 \pm 14.3$ years old $($ mean $\pm \mathrm{SD})$, had $5.3 \pm 2.8$ diagnoses and a CIRS score of $10.3 \pm 5.7 ; 29 \%$ were male. Summary scores of SF-
36 were $42.22 \pm 12.28$ and $50.37 \pm 10.21$ for the physical (PCS) and mental (MCS) components respectively. Figure 1 shows the proportion of each severity level for the 14 domains covered by the CIRS. Due to the small number of subjects, we combined the "extremely severe" CIRS category with the "severe" CIRS category.

Results from the bivariate analyses of the 14 domains of the CIRS with the PCS are shown in Table 1. Significant differences in the PCS among CIRS severity categories ( $\mathrm{p}$ $<0.01$ ) were found in 12 CIRS domains. Among these domains, only 2 subgroups of disease severity could be differentiated according to the PCS in 9 CIRS domains. Three subgroups of disease severity could be differentiated in the 3 remaining CIRS domains (Respiratory, Musculoskeletal and Endocrine).

In the analyses of interactions, only 2 were significant: the Cardiac-Respiratory and the Vascular-Upper gastrointestinal. Details are shown in Table 2.

In the bivariate analyses of the 14 domains of the CIRS with the MCS, a significant difference in MCS was found only between 2 severity subgroups in the Psychiatric domain: no problem/mild/moderate vs. severe $(\mathrm{p}<$ $0.001, \eta^{2}=6.54 \%$ ). Therefore, a multivariate model was not necessary for the mental component.

Results from the multivariate analysis for the PCS are shown in Table 3. The CIRS domains Musculoskeletal, Neurological, and Psychiatric, have an independent direct impact on PCS while the Upper gastrointestinal, Vascular,

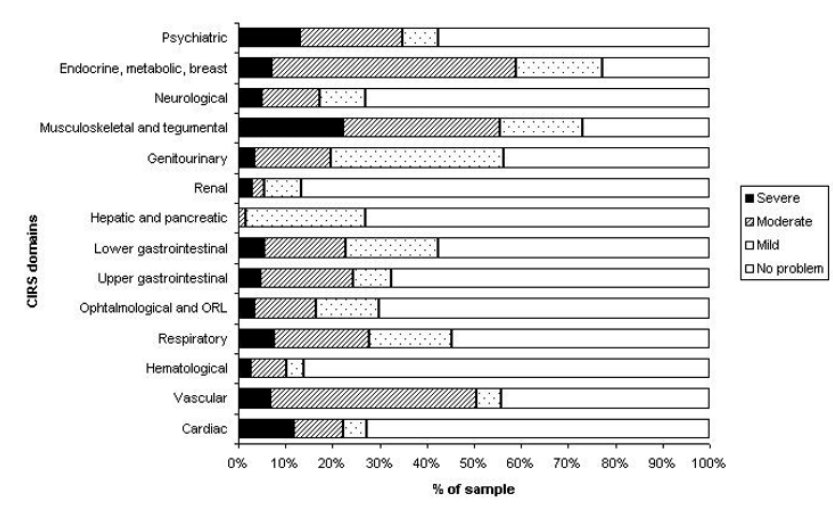

Figure I

Percentage of sample in each severity level. The percentage of sample in each severity level is shown for the 14 domains covered by the CIRS. It should be noted that The CIRS assigns a value from 0 to 4 to determine a severity score for each domain $(0=$ no problem; $I=$ mild; 2 = moderate; $3=$ severe; $4=$ extremely severe). Due to the small number of subjects, we combined the "extremely severe" CIRS category with the "severe" category. 
Table I: Relationship between the physical component summary (PCS) of SF-36 and severity of problems in each domain of the CIRS

\begin{tabular}{|c|c|c|c|}
\hline Degrees of severity by domain of CIRS & PCS (mean \pm sd) & p value & $\eta^{2}$ \\
\hline \multicolumn{4}{|l|}{ Cardiac } \\
\hline No problem/Mild/Moderate & $43.2 \pm 11.8$ & 0.0004 & $5.19 \%$ \\
\hline Severe & $39.2 \pm 12.9$ & & \\
\hline \multicolumn{4}{|l|}{ Vascular } \\
\hline No problem & $46.7 \pm 11.4$ & $<0.0001$ & $10.83 \%$ \\
\hline Mild/Moderate/Severe & $38.6 \pm 11.8$ & & \\
\hline \multicolumn{4}{|l|}{ Haematological } \\
\hline No problem & $42.7 \pm 12.1$ & 0.1221 & $1.01 \%$ \\
\hline Mild/Moderate/Severe & $39.2 \pm 12.9$ & & \\
\hline \multicolumn{4}{|l|}{ Respiratory } \\
\hline No problem/Mild & $44.7 \pm 11.7$ & $<0.0001$ & $12.14 \%$ \\
\hline Moderate & $37.5 \pm 10.9$ & & \\
\hline Severe & $31.2 \pm 12.4$ & & \\
\hline \multicolumn{4}{|l|}{ Ophthalmologic and ORL } \\
\hline No problem/Mild & $43.4 \pm 12.1$ & 0.0013 & $4.32 \%$ \\
\hline Moderate/Severe & $36.5 \pm 11.6$ & & \\
\hline \multicolumn{4}{|l|}{ Upper gastrointestinal } \\
\hline No problem/Mild & $44.3 \pm 11.6$ & $<0.0001$ & $9.17 \%$ \\
\hline Moderate/Severe & $35.7 \pm 12.2$ & & \\
\hline \multicolumn{4}{|l|}{ Lower gastrointestinal } \\
\hline No problem/Mild & $43.4 \pm 12.1$ & 0.0050 & $3.30 \%$ \\
\hline Moderate/Severe & $38.1 \pm 12.2$ & & \\
\hline \multicolumn{4}{|l|}{ Hepatic and pancreatic } \\
\hline No problem & $42.9 \pm 11.8$ & 0.1483 & $0.89 \%$ \\
\hline Mild/Moderate & $40.3 \pm 13.3$ & & \\
\hline \multicolumn{4}{|l|}{ Renal } \\
\hline No problem/Mild & $42.6 \pm 12.2$ & 0.0457 & $1.69 \%$ \\
\hline Moderate/Severe & $35.6 \pm 12.3$ & & \\
\hline \multicolumn{4}{|l|}{ Genitourinary } \\
\hline No problem & $44.5 \pm 12.2$ & 0.0101 & $2.78 \%$ \\
\hline Mild/Moderate/Severe & $40.4 \pm 12.1$ & & \\
\hline \multicolumn{4}{|l|}{ Musculoskeletal } \\
\hline No problem & $48.8 \pm 9.2$ & $<0.0001$ & $16.44 \%$ \\
\hline Mild/Moderate & $42.0 \pm 12.2$ & & \\
\hline Severe & $34.6 \pm 11.2$ & & \\
\hline \multicolumn{4}{|l|}{ Neurological } \\
\hline No problem & $44.5 \pm 11.6$ & $<0.0001$ & $9.72 \%$ \\
\hline Mild/Moderate/Severe & $35.9 \pm 11.9$ & & \\
\hline \multicolumn{4}{|l|}{ Endocrine, metabolic, breast } \\
\hline No problem/Mild & $44.8 \pm 11.6$ & 0.0014 & $5.44 \%$ \\
\hline Moderate & $41.3 \pm 11.9$ & & \\
\hline Severe & $33.9 \pm 14.8$ & & \\
\hline \multicolumn{4}{|l|}{ Psychiatric } \\
\hline No problem/Mild & $44.5 \pm 11.6$ & $<0.0001$ & $6.58 \%$ \\
\hline Moderate/Severe & $37.9 \pm 12.4$ & & \\
\hline
\end{tabular}

Cardiac and Respiratory domains are involved in interactions. The chronic conditions most commonly found in these domains were, respectively: stomach or duodenal ulcers and gastro-oesophageal reflux; hypertension; coronary artery disease and chronic heart failure; chronic obstructive pulmonary disease and asthma. The coefficients in Table 3 allow calculation of an estimate of the PCS for subjects with different problems. For example, for a subject with no problems in the musculoskeletal, neurological and vascular domains and no or mild problems in the psychiatric and upper gastrointestinal domains, the PCS for moderate or severe respiratory problem and severe cardiac problem can be estimated as:

$$
\mathrm{PCS}=54.62+(-3.11)+(-1.85)+(-8.85)=40.8
$$

For subjects with no problems in the musculoskeletal and neurological domains, no or mild problems in the psychiatric and respiratory domains and no severe cardiac problems, the PCS for moderate or severe upper 
Table 2: Significant results of the analysis of interactions among CIRS domains

\begin{tabular}{|c|c|c|c|}
\hline Interaction & PCS (mean \pm sd) & p value & $\eta^{2}$ \\
\hline \multicolumn{4}{|l|}{ Cardiac - Respiratory } \\
\hline Low cardiact and low respiratory $\ddagger$ & $44.8 \pm 11.5$ & $<0.0001$ & $14.99 \%$ \\
\hline Severe cardiac and low respiratory $\ddagger$ & $43.4 \pm 13.4$ & & \\
\hline Low cardiact and high respiratoryई & $38.4 \pm 11.2$ & & \\
\hline Severe cardiac and high respiratory§ & $27.0 \pm 8.0$ & & \\
\hline \multicolumn{4}{|l|}{ Vascular - Upper gastrointestinal } \\
\hline No vascular and low upper gastro $\ddagger$ & $49.2 \pm 9.3$ & $<0.0001$ & $20.12 \%$ \\
\hline Vascular $¥$ and low upper gastro $\ddagger$ & $39.9 \pm 11.7$ & & \\
\hline No vascular and high upper gastro§ & $36.1 \pm 13.5$ & & \\
\hline Vascular $¥$ and high upper gastro§ & $35.5 \pm 11.6$ & & \\
\hline
\end{tabular}

† No problem, mild or moderate problem

$\ddagger$ No problem or mild problem

$\S$ Moderate or severe

$¥$ Mild, moderate or severe

gastrointestinal problem and mild, moderate or severe vascular problem can be estimated as:

$$
\mathrm{PCS}=54.62+(-8.98)+(-6.58)+(+8.08)=47.1
$$

Graphical displays of these interactions are shown in Figures 2 and 3.

\section{Discussion}

In the present study, we have been able to develop a multivariate model relating the physical aspect of HRQOL (as measured by the PCS of the SF-36) and all chronic conditions - grouped by body systems - of patients with multimorbidity, while taking into account the degree of severity of the health impairments evaluated with the CIRS. Although small in size, this is the first study evaluating the impact of systems involvement and their combination on HRQOL.

The summary scales PCS and MCS were chosen to represent HRQOL in this study because they have been shown to be among the most valid SF-36 scales for measuring physical health and mental health, respectively [21]. Also, confidence intervals around individual scores are much smaller for the two summary measures than for the eight scales $(+/-6-7$ points versus $+/-13-32$ points, respectively) [21].

In our multivariate model, all variables have a different weight on the resultant PCS. Impairments in all the CIRS domains that remained in the multivariate model have a negative coefficient (Table 3 ). This means that, according to the model, these impairments have a negative impact on PCS. The impairment with the highest direct impact was severe Musculoskeletal problem reducing PCS by 8 points (Table 3). This latter result is consistent with reports associating musculoskeletal disorders with large losses of HRQOL [22,23]. This is also congruent with the clinical experience of family doctors. Diseases included in the musculoskeletal system are any form of arthritis, chronic low back pain and any rheumatologic or orthopaedic chronic problems.

Table 3: Reduced multivariate model for the physical component summary (PCS) of the SF-36

\begin{tabular}{|c|c|c|c|}
\hline Variable & Parameter Estimate & Standard error & p value \\
\hline Intercept & 54.62 & 1.42 & $<0.0001$ \\
\hline Mild or moderate Musculoskeletal problem (Mus I,2) & -3.77 & 1.53 & 0.0145 \\
\hline Severe Musculoskeletal problem (Mus3,4) & -8.11 & 1.92 & $<0.0001$ \\
\hline Mild, moderate or severe Neurological problem $(\mathrm{Neu} \mathbf{1}, \mathbf{2 , 3 , 4})$ & -3.84 & 1.50 & 0.0111 \\
\hline Moderate or severe Psychiatric problem (Psy2,3,4) & -4.06 & 1.40 & 0.0041 \\
\hline Severe Cardiac problem $($ Car3,4) & -1.85 & 2.83 & $0.5142 \dagger$ \\
\hline Moderate or severe Respiratory problem $(\mathbf{R e s} 2,3,4)$ & -3.11 & 1.62 & $0.0564 \dagger$ \\
\hline Car3,4 and Res2,3,4 & -8.85 & 4.07 & 0.0307 \\
\hline Moderate or severe Upper gastrointestinal problem $(\mathbf{U p g} \mathbf{2 , 3 , 4})$ & -8.98 & 2.47 & 0.0003 \\
\hline Mild, moderate or severe Vascular problem (Vas I, $\mathbf{2 , 3 , 4})$ & -6.58 & 1.49 & $<0.0001$ \\
\hline Upg2,3,4 and Vas $I, 2,3,4$ & +8.08 & 3.10 & 0.0097 \\
\hline
\end{tabular}

$\dagger$ kept in the model because involved in an interaction 


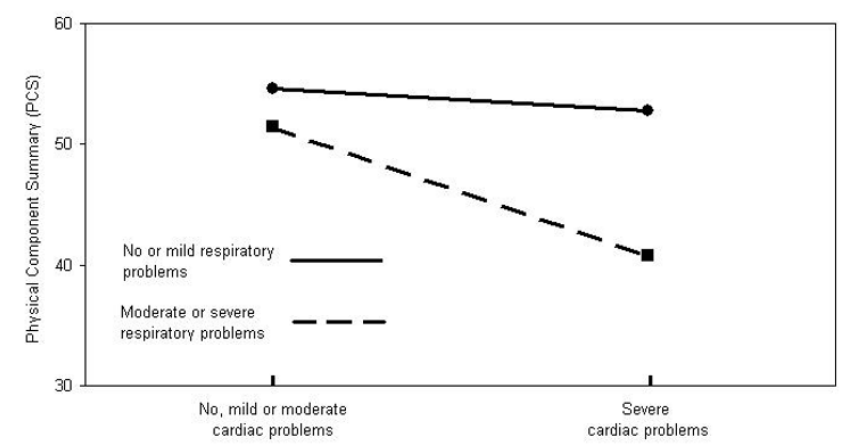

Figure 2

Illustration of the interaction between Cardiac and Respiratory CIRS domains. The figure illustrates the interaction between Cardiac and Respiratory domains for subjects with no problems in the musculoskeletal, neurological and vascular domains and no or mild problems in the psychiatric and upper gastrointestinal domains.

Apart from being additive (the sum of the independent effect of each condition approximates their combined effect), the combined influence of pairs of chronic conditions on the HRQOL can also be synergistic (the combined effect is greater than the sum of the independent effect of each condition) or subtractive (the combined effect is smaller than the sum of the independent effect of each condition) [7-10,24].

We found a synergistic effect between respiratory and cardiac systems which replicates results previously reported by Rijken et al.[10]. They found that patients with cardiovascular disease reported equal levels of PCS as patients with other chronic diseases considered in their study. However, patients with respiratory disease reported worse PCS than patients with other chronic disease, and the combination of cardiovascular and respiratory diseases had a negative synergistic effect on the PCS [10]. Our data confirm the same synergistic effect of these systems on PCS since, in our multivariate model, cardiac problems are not significant, respiratory problems only approach significance and their interaction is highly significant i.e. their combined effect decreases more the PCS than the sum of the independent negative effect of each condition. This is congruent with what we see in our clinical practice. A possible explanation is the common shortness of breath that is present when both systems are involved.

We found a subtractive interaction between moderate or severe Upper gastrointestinal problem and mild, moderate or severe Vascular problem. The independent effect of each condition is quite important in reducing physical functioning, but their combined effect is smaller than the sum of the independent effects (Figure 3 ). We cannot pro-

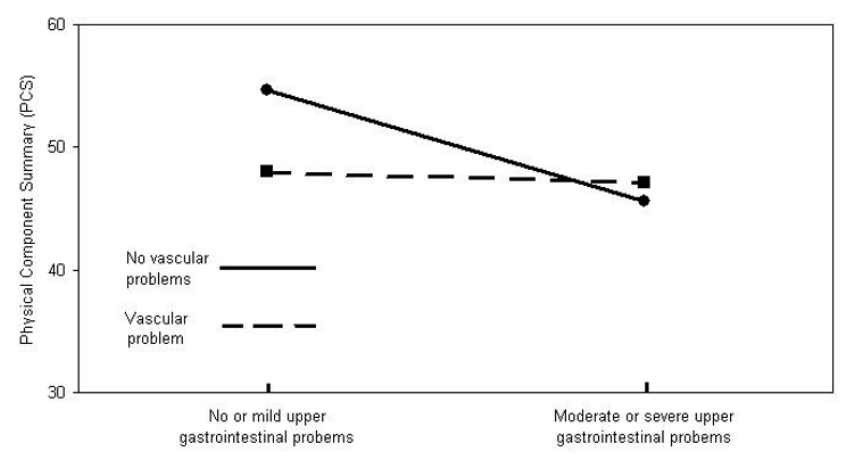

Figure 3

Illustration of the interaction between Upper gastrointestinal and Vascular CIRS domains. The figure illustrates the interaction between Upper gastrointestinal and Vascular CIRS domains for subjects with no problems in the musculoskeletal and neurological domains, no or mild problems in the psychiatric and respiratory domains and no severe cardiac problems.

vide a logical explanation for this finding. This will have to be confirmed and explored in more details.

The literature reports other additive and synergistic interactions of pairs of diseases such as diabetes-hypertension (Endocrine-Vascular domains in the CIRS), diabetes-heart disease (Endocrine-Heart), diabetes-arthritis (EndocrineMusculoskeletal) and arthritis-respiratory disease (Musculoskeletal-Respiratory) that were not found to be significant in our analysis of interactions by CIRS domains [7$10,24]$. Chronic conditions in CIRS domains or interactions not included in the reduced multivariate model should not be interpreted as domains in which health problems do not have an impact on HRQOL or that those interactions are not important for individual patients. It only means that the affected domains and their interactions included in the model are those with a greater impact on the physical functioning in the sample of subjects of this study.

It is worth noting that the Psychiatric domain of the CIRS remained significant in the multivariate model for the physical functioning. Whereas this domain is frequently excluded from studies relating comorbidity or multimorbidity with HRQOL [25], our results highlight its importance in such studies and are in agreement with those reported by Rijken et al.[10].

Rijken et al. also assessed the joint effects of a limited number of somatic chronic diseases and found that, in general, the MCS was not related to any of the diseases evaluated with the exception of a negative effect of thyroid dysfunction [10]. In our study, the bivariate analysis 
showed a significant relationship of the MCS only with the Psychiatric domain. As we evaluated the levels of severity, we found that the significant difference was at the "Severe" level.

Knowledge of the estimates of the relative impact of individual chronic diseases on HRQOL is important for decision-makers to better plan and allocate resources for research, training and health care [4]. For the primary care physician, for whom multimorbidity implies a list of health problems, the knowledge of specific systems or their combinations that have a greater impact on the patient's HRQOL is essential for individual patient care and can be helpful to establish priorities in management decisions [24]. Given that patients with multimorbidity represent the majority in primary care, this knowledge is also important for public health purposes.

This study was based on the analysis of an existing database, and its main limitation is the relatively small sample of subjects. This limitation is directly related to the effect of the intentional sampling process to recruit patients that represented five CIRS groups, each with different levels of multimorbidity. This sampling process by itself was not expected to affect the relationships in our analyses but produced its effect through the size of the sample we obtained. The number of subjects per severity level varied considerably. This forced us to group subjects with problems of different levels of severity. Also, a number of diseases within the CIRS domains may not be represented. A bigger sample would have given more power to the statistical analyses and any application of the multivariate model at its present state should be made with caution. Another limitation of the study is that the list of diagnosis was based on chart review of all diagnoses already made by the primary care physician and no attempt was made to assess whether the diagnoses were correct. This method has the limitation that conditions frequently misdiagnosed such as asthma and chronic obstructive pulmonary disease, or frequently undiagnosed such as mental disorders were not verified. Due to all the limitations listed above, we consider that any generalizability of the model at its present state should be made with caution. It might be applicable only to the primary care clientele of our region.

Unlike previous studies, we did not analyze the combined effects from a pre-determined list of diseases; instead, we included in the corresponding CIRS domains all the chronic conditions found in the subjects. This approach is pragmatic and directly in line with clinical practice. Nevertheless, the combined influence of more than two chronic conditions pertaining to more than two systems on the HRQOL can be expected to be more complex than that of a pair of diseases and consequently, caution is mandatory in the interpretation of the study results.

\section{Conclusion}

With this study we have demonstrated the feasibility of developing a multivariate model for the physical functioning aspect of the HRQOL applicable to patients with multimorbidity that takes into account all the chronic diseases (grouped by organ domains) and their level of severity, while controlling for age, education, perceived social support and economic status. Simple disease count as a measure of multimorbidity has a weak correlation with HRQOL; grouping diseases by organ domains which take into account disease severity is a practical alternative to consider individually each condition and its severity. Vascular, Upper gastrointestinal and Musculoskeletal systems have strong negative effects on HRQOL. Among combinations of systems, the respiratory and cardiac combination is of particular concern because of a synergistic negative effect. This study paves the way for a future study with a bigger sample that could yield a model of wider generalizability.

\section{Competing interests}

The author(s) declare that they have no competing interests.

\section{Authors' contributions}

MF participated in the conception and design of the study, supervised data collection and analysis and drafted the manuscript. M-FD participated in the conception and design of the study, performed the statistical analysis and helped draft the manuscript. $\mathrm{CH}$ participated in the design of the study critically reviewed the manuscript. HS participated in data analysis and critically reviewed the manuscript. JA participated in the data analysis and helped draft the manuscript. All authors gave their final approval of the version of the manuscript submitted for publication.

\section{Acknowledgements}

Sources of support: Fonds de la Recherche en Santé du Québec (Grant number: 24300-2028) and Pfizer Canada (Independent Research Grant).

\section{References}

I. Grumback K: Chronic illness, comorbidities, and the need for medical generalism. Ann Fam Med 2003, I:4-7.

2. Fortin M, Bravo G, Hudon C, Vanasse A, Lapointe L: Prevalence of multimorbidity among adults seen in family practice. Ann Fam Med 2005, 3:223-228.

3. Stange KC: The paradox of the parts and the whole in understanding and improving general practice. Int J Qual Health Care 2002, 14:267-268.

4. Sprangers MA, de Regt EB, Andries F, van Agt HM, Bijl RV, de Boer JB, Foets M, Hoeymans N, Jacobs AE, Kempen GI, Miedema HS, Tijhuis MA, de Haes HC: Which chronic conditions are associated with better or poorer quality of life? J Clin Epidemiol 2000, 53:895-907. 
5. McDowell I, Newell C: Measuring health. A guide to rating scales and questionnaires. 2nd ed. New York, Oxford University Press; 1996.

6. Fortin M, Bravo G, Hudon C, Lapointe L, Almirall J, Dubois MF, Vanasse A: Relationship between multimorbidity and healthrelated quality of life of patients in primary care. Qual Life Res 2006, I5:83-9|.

7. Wee HL, Cheung YB, Li SC, Fong KY, Thumboo J: The impact of diabetes mellitus and other chronic medical conditions on health-related Quality of Life: is the whole greater than the sum of its parts? Health Qual Life Outcomes 2005, 3:2.

8. Ettinger WH, Davis MA, Neuhaus JM, Mallon KP: Long-term physical functioning in persons with knee osteoarthritis from NHANES. I: Effects of comorbid medical conditions. J Clin Epidemiol 1994, 47:809-8I5.

9. Oldridge NB, Stump TE, Nothwehr FK, Clark DO: Prevalence and outcomes of comorbid metabolic and cardiovascular conditions in middle- and older-age adults. J Clin Epidemiol 200I, 54:928-934.

10. Rijken M, van Kerkhof M, Dekker J, Schellevis FG: Comorbidity of chronic diseases: effects of disease pairs on physical and mental functioning. Qual Life Res 2005, 14:45-55.

II. Linn BS, Linn MW, Gurel L: Cumulative illness rating scale. J Am Geriatr Soc 1968, 16:622-626.

12. Miller EA, Towers A: A manual of guidelines for scoring the cumulative illness rating scale for geriatrics (CIRS-G). University of Pittsburgh; 1991.

13. Harboun M, Ankri J: Comorbidity indexes: review of the literature and application to studies of elderly population. Rev Epidemiol Sante Publique 200 I, 49(3):287-298.

14. de Groot V, Beckerman H, Lankhorst G], Bouter LM: How to measure comorbidity. a critical review of available methods. J Clin Epidemiol 2003, 56:221-229.

15. Fortin M, Hudon C, Dubois MF, Almirall J, Lapointe L, Soubhi H: Comparative assessment of three different indices of multimorbidity for studies on health-related quality of life. Health Qual Life Outcomes 2005, 3:74.

16. Fortin M, Bravo G, Hudon C, Lapointe L, Dubois MF, Almirall J: Relationship between psychological distress and multimorbidity of patients in family practice. Ann Fam Med 2006, 4:417-422.

17. Miller MD, Paradis CF, Houck PR, Mazumdar S, Stack JA, Rifai AH, Mulsant B, Reynolds CF 3rd: Rating chronic medical illness burden in geropsychiatric practice and research: application of the Cumulative IIIness Rating Scale. Psychiatry Res 1992, 4I:237-248.

18. Hudon C, Fortin M, Vanasse A: Cumulative Illness Rating Scale was a reliable and valid index in a family practice context. I Clin Epidemiol 2005, 58:603-608.

19. Ware JE, Sherbourne CD: The MOS 36-item short-form health survey (SF-36). I. Conceptual framework and item selection. Med Care 1992, 30:473-483.

20. Cutrona CE, Russell DW: The provisions of social support and adaptation to stress. Advances in Personal Relationships 1987, I:37-67.

21. Ware JE]: SF-36® Health Survey Update. [http://www.sf-36.org/ tools/sf36.shtml].

22. Rabenda V, Manette C, Lemmens R, Mariani AM, Struvay N, Reginster JY: Prevalence and impact of osteoarthritis and osteoporosis on health-related quality of life among active subjects. Aging Clin Exp Res 2007, 19:55-60.

23. Saarni SI, Harkanen T, Sintonen H, Suvisaari J, Koskinen S, Aromaa A, Lonnqvist J: The impact of 29 chronic conditions on healthrelated quality of life: a general population survey in Finland using I5D and EQ-5D. Qual Life Res 2006, I 5(8): | 403-I4I4.

24. Fried LP, Bandeen-Roche K, Kasper JD, Guralnik JM: Association of comorbidity with disability in older women: the Women's Health and Aging Study. J Clin Epidemiol 1999, 52:27-37.

25. Fortin M, Lapointe L, Hudon C, Ntetu AL, Maltais D, Vanasse A: Multimorbidity and quality of life in primary care: $A$ systematic review. Health Qual Life Outcomes 2004, 2:5I.

\section{Publish with Biomed Central and every} scientist can read your work free of charge

"BioMed Central will be the most significant development for disseminating the results of biomedical research in our lifetime. "

Sir Paul Nurse, Cancer Research UK

Your research papers will be:

- available free of charge to the entire biomedical community

- peer reviewed and published immediately upon acceptance

- cited in PubMed and archived on PubMed Central

- yours - you keep the copyright

Submit your manuscript here:

http://www.biomedcentral.com/info/publishing_adv.asp
BioMedcentral 